\title{
5 Years experience of Columbus Ground Segment Operations
}

\author{
Dr. Osvaldo Peinado ${ }^{1}$ \\ Ground Operations Manager \\ German Space Operations Center (GSOC) \\ Oberpfaffenhofen \\ 82234 Wessling \\ Germany
}

The Columbus Ground segment is a very complex system with connections to USA (NASA), Russia (RSA), ATV-CC and many user centers across Europe. Because of the many delays in the launch of the Columbus module to the ISS, the ground segment was used to support other activities between the ISS and centers in Europe, like the interim utilization, Eneide and Astrolab missions, among parallel activities such as simulations.

This decision provided a good opportunity to test the systems and be prepared for the Columbus mission. On the other side now, after 2 years of Columbus operations, Col-CC is facing the issues that almost all the subsystems are running out of maintenance contracts, and in addition, the hardware and operating systems are aging. Some of the subsystems need an urgent update in order to ensure the required support to Columbus operations. That results in a new challenge of testing in parallel the new systems without affecting operations. It is important to note that Col-CC does not have a testing facility for most of the subsystem and is under a very complicated decision-making and financial structure that makes the maintenance of the current functionalities extremely complex.

All of these factors combined with the budget and man power limitations makes the successful short history of the operations and the already start of the parallel migration an interesting defiance.

\section{Introduction}

ESA's Board of Directors approved the Columbus program in 1985. From then on, numerous studies and proposals were made. The structure used is based on the Multi-Purpose Logistics Module, a module built for NASA by Thales Alenia Space, which was designed for ten years of operation. The module is controlled by the Columbus Control Center, located at the German Space Operations Center (GSOC), part of the German Aerospace Center (DLR) in Oberpfaffenhofen near Munich, Germany.

The name of the Columbus module was chosen because it was planned to launch the European Laboratory 500 years after Christopher Columbus arrived to America, which means 12 of October of 1992. Actually, it was launched aboard the Space Shuttle Atlantis on February 7, 2008 on flight STS-122. That was an effective delay of 16 years. There were many causes for such an unbelievable delay, mainly ESA political decisions and budget cuts, redesign, aging of hardware and software and the shuttle launch delays after the Columbia accident.

Columbus is a science laboratory that is part of the International Space Station (ISS) and is the largest single contribution to the ISS made by the European Space Agency (ESA). The laboratory is a cylindrical module with two end cones. It is $4,477 \mathrm{~mm}(15 \mathrm{ft})$ in external diameter and $6,871 \mathrm{~mm}(23 \mathrm{ft})$ in overall length, excluding the projecting external experiment racks. Its shape is very similar to that of the Multi-Purpose Logistics Modules, since both were designed to fit in the cargo bay of a Space Shuttle orbiter. The starboard end cone contains most of the laboratory's on-board computers. The port end cone contains the Common Berthing Mechanism.

Columbus is not only the European module connected to the ISS; Columbus implies a complex new international infrastructure across Europe as shown in figure 1.

\footnotetext{
${ }^{1}$ Ground Operations Manager, Communications and Ground Stations, DLR - Muenchenerstr. 20 - 82234 Wessling, Germany, non AIAA Member.
} 
The distributed Columbus ground segment presents a huge logistical challenge. In order to reduce the operational costs but at the same time to meet the requirements of the users, a special team and automated processes were implemented. One difference between Col-CC and NASA or other space agencies, is that at Col-CC the subsystems only have office hours support (8/5), that means in order to bring support to the 24/7 operations of Columbus, the Ground control team (GCT) must assume additional responsibilities and tasks than a normal ground control team.

The GCT consist of two positions on console 24/7 - the Ground Controller (GSOC-GC) and System controller (GSOC-Syscon) - and 3 other positions that are 8/5 but also on call or, in critical situations, also on-site. These off line positions are the ground operations planner (GOP), the subsystems engineers (SSE) and the ground operations manager (GOM).

In this paper a little history of the Columbus control center and its complex distributed network will be presented, as well of the support of other missions, description of the critical situations and the many functions of the Ground Control Team.

\section{Previous missions support}

During the 16 year delay, hundreds of documents with specifications, test procedures, simulations scenarios and network design were generated. After many updates and re-design, finally in 2004, the IGS network shown in figure 1 was deployed, the servers for the SAN (Storage Area Network) were installed, the software of most of the subsystems passed the SAT (site acceptance test) and the personnel to be on console were trained.

All of this was accomplished using simulators. The first IT (interface test) with the USOCs and IP were successfully carried out, but Columbus was still on earth. The ground segment made all the system tests, load tests, SVT tests, etc, but it was not used under real conditions, with real data and users. The 5 Columbus control rooms and 4 of the 8 support rooms were refurbished but never used, except the main control rooms, K4 and GCOR (Ground Control Operations Room) for testing activities and K3 and BuGOR (Backup Ground Operations rooms) for stand alone, internal simulations.

For this reason it was decided to use Col-CC, and specially the ground segment to support other ESA missions and be prepared for Columbus. During the so called interim utilization the first 2 previous Columbus missions were supported, ${ }^{\text {st }}$ Eneide and $2^{\text {nd }}$ Astrolab as described below.

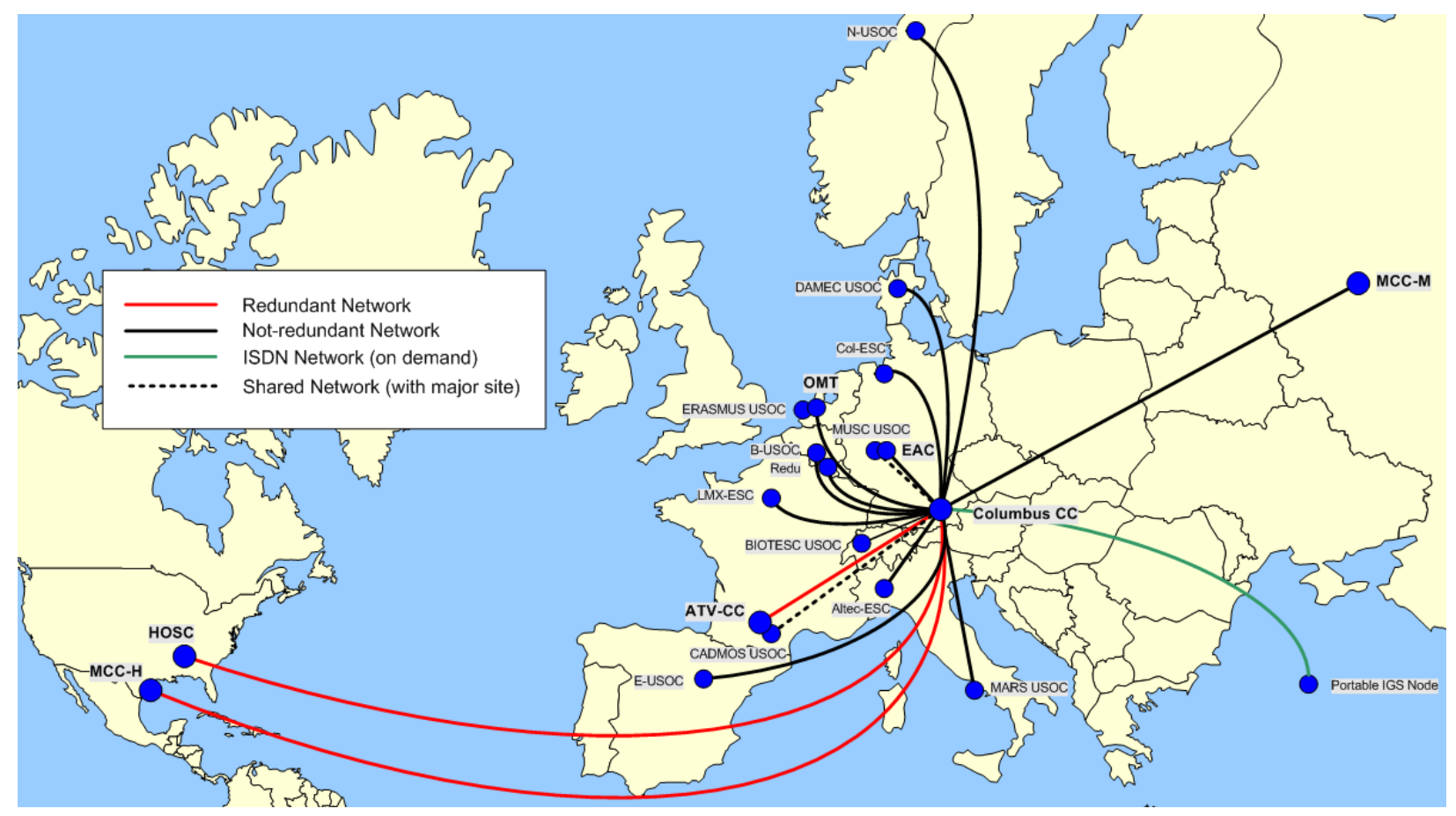

Figure 1 : International distribution of the Columbus Ground Segment 


\section{A. Mission Eneide}

The European Space Agency Italian Astronaut, Roberto Vittori, launched with Expedition 11 on the Soyuz TMA-6 spacecraft and returned April 24, 2005 with Expedition 10 on Soyuz TMA-5 under an agreement between the Russian Rosaviakosmos, the Italian Space Agency, ASI and ESA. During his stay aboard ISS he worked alongside the resident crew overseeing four European scientific experiments.

This mission only lasted 10 days, but was the first opportunity to test equipment, software, communications and the readiness of the teams to carry out a real manned space mission.

For the ground segment, the first complicated task was to connect the University of Lazio, Rome (LUC- Lazio User center) sending video and audio to a non IGS node. This was solved using soft keysets connected to an ISDN BRI line directly to Col-CC. Video was also converted at Col-CC (bandwidth reduced), and trough extra OPS (TM) and OPS SUP ISDN PRI lines sent to Italy. The only issue was during the testing phase. It was found that the ISDN standards in Europe changed the standard parameters among the countries. ISDN was initialy developed in Germany and the standard defined, but the different companies in each country made their own adjustements and that required additional testing efforts in order to get the voice system working, by adapting the parameters to the real standard.

It was also the first real use of the ATM and ISDN connections to JSC, Moscow, EAC (European Astronaut Center) and others USOCs in Europe. During this mission 24/7 support was not performed, but the GCT and especially the SSE got a good training in real time operations.

\section{B. Mission Astrolab}

The Astrolab Mission was Europe's first long-duration mission to the International Space Station (ISS). For the journey to the ISS, German ESA astronaut Thomas Reiter joined the crew of the STS-121 mission, which was launched on Space Shuttle Discovery in 4 July 2006. The mission was supported by a tri-lateral understanding between ESA, Roscosmos and NASA.

The Astrolab Mission marks the first time that a European scientific program has been assembled for a longduration mission. The program was predominantly from scientific institutions across Europe, including experiments in human physiology, biology, physics and radiation dosimetry. Other activities were focused on technology demonstrations, industrial experiments and education, extensive testing of the PR connections from the COL-CC ground segment (GS) to the different TV stations. On this mission the first ESA astronaut undertook a spacewalk or Extra Vehicular Activity (EVA) from the ISS with the corresponding PR support from the GS.

Space Shuttle Discovery also took three ESA-developed experiment facilities and devices to the International Space Station, which for the GS implies connections to Marshall in order to get the high rate data from the experiments.

Col- CC was the hub of European activity during the mission, with tasks such as:

- Monitoring and coordinating Reiter's activities

- Coordinating with Russian and American mission control centers

- Coordinating with the European Astronaut Center, in Cologne, Germany

- Coordinating with User Support and Operations Centers (USOCs) throughout Europe

- First time having 16/5 ground and flight team support, testing the shift work and handover procedures

- Coordination between flight and ground control teams for support of real time, test and simulation activities in parallel

The Astrolab Mission provided Europe with invaluable experience of long-term scientific utilization of the ISS in advance of the launch of the European Columbus laboratory. It was a very good opportunity to test all the connections to the IP and USOCs, the first constraints caused by the use of ISDN lines was also understood by the users. Originally it was planned to have ATM connections for everybody in the IGS network and the ISDN lines only as backup, but because of budget cuts, ISDN lines became the only ones available for the USOCs. There were strong reductions in the bandwidth available, especially for OPS-SUP usage, because the video was sent using the same network as the OPS SUP tools.

COL-CC video SSE reduced the MPEG 2 stream to $1.3 \mathrm{MB}$ (with overhead) to be sent via ISDN PRI (2 MB). That abated of course the video quality, but the real problem was during the burst updating of OSTPV and others tools. During those updates, the video lost more quality and all the tools became very slow. The solution was to limit the usage of OPS support LAN in an operational way. E.g. to not use video and OSTPV at the same time in the same network. A traffic prioritization in ISDN lines was not logical. 
During this mission the lack of SSE support (8/5) during the 16/5 operations, generated the necessity to assign more tasks to the GCs and Syscons in order to solve problems based on procedures. Not operational procedures, but recovery ones, were written down, generating an intensive interaction between SSE and people on console.

The handover became a very important piece for operations, especially after weekends. The console logs became a central tool to follow different problems; it was a learning curve for the people on both teams to properly use them.

The system started to have real workload, especially high rate data from Marshal, causing a lot of modifications in the software and network configuration parameters that didn't appear during the test phase. Long use of the applications and hardware shows issues with memory usage, software configuration and also hardware failures.

All of these many issues and their resolutions immensely helped to get the software, hardware and people prepared for the Columbus mission.

\section{Mission Columbus}

As mentioned, after the many initial delays, new problems with the launch produced further delays in the start to the mission. In November 2007, Columbus was moved out of the KSC Space Station Processing Facility, and installed into the payload bay of the Atlantis orbiter for launch on ISS assembly flight 1E. During cryo-filling of the space shuttle External Tank, prior to the first launch attempt on December 6, 2007, two of four LH2 ECO sensors failed a test, resulting in a next launch attempt set for Sunday December 9, 2007. This launch attempt was scrubbed when one of the ECO sensors again failed during fuelling. The ECO sensors external connector was changed on the space shuttle external tank, causing a two month delay in the launch. Columbus was finally launched successfully on the third attempt at 2:45pm EST, February 7, 2008.

The Columbus mission is the second European manned long term mission. Many teams and centers distributed across Europe, USA, Russia, Canada and Japan are working together. Inside Col-CC are also many teams that cover different aspects of the mission as is described below. The administration is a very complicated combination between ESA, Astrium, DLR and many other companies. That makes the decision process really slow.

The Control Center is the direct link to Columbus in orbit. The center's main functions are to command and control the Columbus laboratory systems, to coordinate operations of the European payloads on board the ISS and to operate the European ground communications network. Since the Columbus laboratory itself hosts non-European experiments, such as US payload facilities, decisions taken, for example, changes in scheduling are coordinated with the ISS international partners. For this reason the Columbus Control Center is connected to the ISS Mission Control Center at the Johnson Space Center in Houston, the Huntsville Operations Support Center in Huntsville, Alabama, and to the ISS Mission Control Center in Moscow.

\section{Columbus Ground Control team}

Because of the reduced budget that ESA disposed for the GCT, the functions and responsibilities of the positions in the GCT are clearly more complex that a normal flight or ground controller. For this reason the 2 GCT console positions were allocated in a separate control room with additional monitoring and commanding possibilities than a standard position. For example, a flight controller has 4 to 8 screens to monitor; a ground controller has 15 to 20 screens. Because the GCT is not in the same room and doing an "invisible Job" in Col-CC is quite often only mentioned the FCT and forgot the key positions of GC and Syscon as a part of the operations team.

The fact that they are sitting in another room, 2 floors above, and are only heard on the voice loops or seen in case of failure, makes the FCT feel as though the GCT is another team. The reality is there is only one team, divided into 2 control rooms, operating simultaneously due to space limitations with different responsibilities and tasks. The GCT is also supporting real time, test and simulation activities at the same time. The GCT alone is also supporting the same 3 activities of the ATV project, for these reasons also, a physical separation on the positions is also needed, in order not to interfere with the real time Columbus operations.

One of the big differences with other space control centers or missions is that the SSE are only available 8/5 and the GCT support operations 24/7, which means that the GC/syscon must to take actions in case of failure outside of office hours. For critical situations SSE are available on-site or on call depending of the severity of the situation, but it is only in reduced period of time and a few times a year. The GOMs were always on-call, now due to DLR internal laws the GOM support must be reduced.

At Col-CC the support is divided in 3 levels: 
1. Level 1 - This encompasses support to nominal, scheduled real time simulation and test operations 24 hours per day, 7 days per week, according to the specific mission requirements and established, approved procedures by the GSOC GC and the GSOC SYSCON (these are the call names of the two positions). This includes the performance of scheduled preventive maintenance. Additionally, Level 1 support encompasses processing of anomalies.

Specifically, the GCT receives problem reports from ESA GS users. They perform initial troubleshooting and initiate corrective actions or implement work arounds utilizing established, approved procedures. Anomalies that are not solvable at Level 1 are escalated to Level 2 and tracked in the GSOC Discrepancy Report system. The GSOC GC is the primary point of contact for ESA GS related issues and anomalies. A GOM is always on call, and many times the decision to escalate a problem to level 2 is taken by the GOM, previous information exchange with the GC/syscon on console and in some cases with the corresponding SSE and flight director.

2. Level $\mathbf{2}$ - This is the first escalation level of anomaly resolution. Errors and anomalies in the system which are tracked, but not solved by the operations staff in Level 1 are escalated to the Subsystem Engineers of the affected subsystem for further investigation. Implementation of corrective actions, including possible hardware replacement, are coordinated with the GCT and performed in such a way as to minimize impact on ongoing operations. If the problem can not be solved by the subsystem expert, the failure resolution will be escalated to Level 3 .

Many of the level 2 issues must be resolved ad hoc or a work around must be implemented in order to guarantee operations. These kinds of decisions are taken by the SSE with GOM approval.

In event of conflicts between Columbus and ATV, if the problem occurs outside office hours, the GOM takes the decisions. The following working day, the priority of the projects and the decisions are discussed with the ESA authorities. Up until now all the conflicts solved by the GOM were successful and no problems or extra costs to any project were caused; furthermore, no GOM decision was retracted. Because the majority of Col-CC and IOT system implementations have redundancy, most anomalies only affect redundancy and not functionality or service interruption as is described below

3. Level 3 - This encompasses vendor support to isolate, identify and correct problems that could not be corrected in Level 2. At this level the changes require more time and money and are an off line task. During the meantime, a work around must be implemented until the vendor solution is released, tested and can be used in the operational environment.

\section{Positions in the GCT}

4. GOM: The GOM has the overall responsibility for GC service coordination and provisions, also the responsibility over the Interfaces to external facilities and users and team Management. In critical situations, the GOM is the central point of contact for making decisions and resolving conflicts between the FD of the different projects (e.g., Columbus and ATV); there are only 2 GOMs at Col-CC.

5. GOP: The Ground Operations Planner's key responsibility is to coordinate and schedule resources for all activities and provide the GOM with background information of requested services for potential scheduling conflicts. The GOP requires general knowledge of the Col-CC subsystems to interact with external sites and the ColCC subsystem engineers in addition to the GCT to analyze and arrange the requested support. There are also only 2 GOPs

6. GC: The GSOC GC serves as the single POC for all execution activities regarding the Columbus facility and Interconnection Ground Subnetwork (IGS) operations. Tasks include the configuration, monitoring and coordination of communications network and restoration activities in response to ground segment problems. Today 9 GCs are available, not enough compared to the 14 persons or more for each FCT position. The GSOC-GC primary interactions are with the International Partners (NASA, RSA) and ATV control centers GC position, the ESA GS user/engineering centers, and the Flight Control Teams (FCTs).

In the event that the GSOC-GC is not able to rectify a service outage, procedures are utilized to coordinate the restoration of service by coordinating the activity with the Col-CC maintenance team and providing a status to affected external sites and FCTs. Both GC and Syscon positions support the new members to the FCT with a teaching and guidance task, independent of normal technical support during simulations to the Sim team.

7. GSOC SYSCON: the GSOC System Controller is responsible for preparation of the Columbus internal systems for scheduled activities, especially the DaSS and MCS subsystems. In the event that the GC is not available short term, the SYSCON has the knowledge and training to support the GC position for external partner interactions. The 
GSOC SYSCON works closely with the subsystem engineers to ensure that any service changes are documented and the GCT procedures are updated and validated. Other tasks of the syscon position are to support the FC in the control room in the event of wrong usage of the software or simple hardware failure. Only 7 certified Syscons are working at Col-CC.

8. SSE (Subsystem Engineer): The subsystems engineers are responsible for the maintenance and correct operations of their own subsystem. The SSEs have a strong interface to the GOM, GOP, GC and Syscon, in case of changes, updated or preventive / corrective maintenance of their system. The SSE is also actively involved in the upgrade of their software and the underlying operating system and also the hardware where the corresponding system is running. Additionally, a SSE is in charge of archiving the relevant data produced by the subsystem and also ensuring that the GCT procedures up to date. There are around 45 SSE working in Columbus, some of them additionally support satellite missions and the shared resources of GSOC.

\section{E. Ground and flight control team}

The integrated Columbus Control Center Flight Control Team is a joint DLR and EADS Astrium team. This mission control service is provided as part of the overall end-to-end operations service delivered by EADS Astrium as the ISS Industrial Operator. The Flight Control Team is led by DLR flight directors and is under the overall supervision of an ESA Mission Director based at DLR Oberpfaffenhofen. The GCT is led by the GOMs and supports the ESA Mission Director. The Col-CC operations teams provide 7 days/week, 24 hours/day operations support for the Columbus mission. The GCTs alone provide 24/7 support to the ATV project. Thereafter, the Col-CC operations are tailored to the payload operations needs.

The communications network established to accomplish these tasks is the Manned Spaceflight and Microgravity Ground Segment (MSM-GS or ESA GS). It consists of networking, processing, storage and communications equipment for data, voice and video products located at the Col-CC and at other control centers, management facilities, International Partners (IPs), Engineering Support Centers (ESCs) and User Support Operations Centers (USOCs). There is also a Simulations team, a training team, an EST (engineering support team), an EPT (European planning team) as well as an ESA collocated team at Col-CC. Each of them has their own support rooms which are all assisted by the GCT simultaneously with the other activities.

\section{F. Support to USOCs}

The Columbus Control Center is also responsible for providing the Ground Segment Services for all European manned space flight activities. This includes connecting the User Support Operations Centers (USOCs) with the Columbus Control Center, and routing data for the Automated Transfer Vehicles (ATVs) to the ATV Control Center located in Toulouse. ESA uses the ATVs to bring supplies and science experiments to the ISS. The Columbus Control Center „supervises the ISS-based tasks of the unmanned European space transporter ATV”.

The USOCs are based in national centers distributed throughout Europe and are responsible for the specific operation of the ESA payload and experiment facilities within the Columbus laboratory. At these centers, scientific investigators can monitor, or be linked to their experiments. Currently there are USOC centers based in Germany, France, Spain, Italy, Norway, Switzerland, Denmark, Belgium and in the Netherlands. Many of these centers also have connections to Universities and other research centers within Europe (UHB). Sometimes these centers also share experiments, which entail configuration changes in the network for the data distribution and security.

The Columbus Control Center is responsible for distributing data to the USOCs and receiving information from them such as requests for resources and reconfiguration of Columbus systems in support of experiments and payload facility operations. Such information is fed into the mission planning process that generates timelines for flight controllers and astronauts. The Columbus Control Center is also linked to the European Astronaut Center (EAC) in Cologne, which is responsible for medical support, monitoring, and safety of ESA astronauts during missions.

With regards to the USOC payload operations, the FCT support with commanding and controlling the systems of the Columbus laboratory, Col-CC ensures that astronauts working within Columbus have a safe and comfortable environment in which to work and that the payload facilities have the necessary systems support in order to function properly. This includes monitoring and configuring, by remote command, the life support systems, which maintain air quality, and the power supply to experiment facilities, as well as systems for removal of heat from experiment facilities. European and non-European astronaut activities inside Columbus are monitored and coordinated from the Columbus Control Center. The Center also holds overall responsibility for safety in the Columbus laboratory under the overall authority of the ISS Mission Control Center in Houston, Texas. The Columbus Control Center reacts to any changes during the mission, coordinating decisions and establishing priorities in the event that a change should interfere with the European experiments inside Columbus. 


\section{G. ATV mission support}

The Automated Transfer Vehicle or ATV is an expendable, unmanned resupply spacecraft developed by the European Space Agency (ESA). ATVs are designed to supply the International Space Station (ISS) with propellant, water, air, payload and experiments. In addition, ATVs can reboot the station into a higher orbit.

The first ATV, Jules Verne, was launched on the $9^{\text {th of }}$ March 2008. ESA has already contracted suppliers to produce four more to be flown until 2015. The second ATV Johannes Klepler must be tested. Therefore, the entire control center in Toulouse was refurbished and many of the systems were updated. This entirely new configuration is now being tested with intense GCT support from Col-CC. JIS (Joint integrated simulations) are planned for 2010 and the first overlaps with Columbus SIMs were identified and communicated to ESA in order to avoid conflicts.

During the first ATV mission, may be it was coincidence, but almost every JIS was overlapped with a European simulation. This situation produces enormous effort from the GCT in order to support these activities in parallel with real time operations. Due to the shared resources, both simulations used the same voice loops, which generated conflicts. For the second ATV mission, Col-CC has advised ESA of possible conflicts. In any event, the GCT will no longer support 2 simulations in parallel.

One of the biggest problems that occurred during the first ATV mission was that only the GCT supported both missions. The FCT practically was not aware of the ATV mission. During the issues described in the next section, it was very difficult for the GOM to coordinate and solve problems with the ATV FD, the Col-CC FD, Moscow FD and NASA FD, especially because the two first FD did not talk to each other. For the second mission and beyond, the GCT is informing and involving the FCT as much as possible in order to avoid conflicts during future missions.

\section{Ground Segment Effectiveness}

It must be explained that the stability of the systems and communication between the teams and the understanding of the systems by the FCT were highly improved during the almost 2 years of Columbus mission.

The number of issues was also greatly reduced. Almost $95 \%$ of the severe problems occurred during the first 1.5 years of operations.

Most of the problems were caused by the MCS, the SAN, the Voice and unexpected problems, such as a Digger cutting cables off the ground. Almost all the problems happened during the night or during the weekend, but not during critical situations where SSE on call support was available, making the problems even worse.

The GOMs were the ones making the difficult decisions and taking the risks, luckily always with great success and support from the GCT. It is also very important to note, that data never was lost, and the redundancy worked perfectly, always allowing uninterrupted operations, in spite of the problems and work arounds. The support of the SSE was unconditional and everyone in the team was helping during the nights or weekends, also not being on call, but staying and coming to Col-CC for as long as was needed. The key to the success of the GCT is commitment and disposition of each member of the team in order to solve the problems, no matter when and how long it takes.

Few minutes before the Columbus docking maneuver one of worst issues occurred. The entire team was ready for the Columbus activation when a Digger working in the Galileo (European GPS) building located across the street opened a hole in the street in the wrong place and cut through all of the main communication cables used for the Columbus Project. In the Columbus Project the OPS (operations) and OPS SUP (operations support) networks are considered critical and are redundant. These networks are going out from the building in different directions and connected to nodes in different cities as well the telephone lines in order to warranty the redundancy.

During this accident, the monitoring system shows immediately the lost of the network and all the applications fail over to the backup. Nobody, the FCT and all the press sitting at Col-CC noticed that we had a severe problem there. The monitoring system is located only in the GCOR. Only some people on console realized that internet (the office network) stop working, but all the operational systems continue working without any interruption. Luckily all the SSE were present and immediately started to check if everything continues nominal.

After this incident an old rule was reapplied at DLR and now no constructions works are allowed in the whole areal during critical operations at GSOC.

Another big issue happened two times during activation or deactivation of Columbus moving payloads between the space shuttle and the ISS. In both cases happened also during the night where no SSE was available. The first time was a combined SAN - MCS failure and the second time was a MCS double failure. In both cases after shortly analyze of the situation with the GC on console and the responsible SSE (called by the GOM and coming to Col-CC not being on duty) it was decided to move the whole FCT to the back up control room (K11). The real time syscon and SSE prepared and checked everything very fast and with a big cooperation of the FCT team the movement was 
carried out. The FCT from K4, the main control room, was moved to K11into another Building. The transition time was 15 minutes, but the systems were configured in a way that no single bit was lost. The system change was coordinated between FCT and GCT leaving all in hot stand by, till the FD gave the GO for the activation of the "new" control room. These decisions were heavily discussed by ESA GS, just because it was not understood the severity of the situation and that no data or commanding capability was lost. After these 2 incidents, a new rule was implemented, saying that the backup control room must be in hot redundancy during critical situations. The rest of the time can stay in cold redundancy (24 hours activation time) as usual

Being in this new situation, the problems happened a $3^{\text {rd }}$ time, but because of the hot redundancy applied and the "training" of the FCT and GCT, the displacement was done almost as a standard procedure without any inconvenience. The SAN and MCS SSE together with the GOM decided to implement a complete new configuration for both subsystems. That decision found a hard resistance from ESA GS; till it was finally implemented, trough the GOM pressure and demonstrated that it worked. Since the new so called "LUN configuration" was implemented, those issues never happened again and also any other. After the successful implementation and operational stability of this solution, some ideas come up to apply this configuration for the whole SAN, but that is not feasible and not needed. The other subsystems never had this kind of problems. Nowadays ESA GS learnt that the Col-CC decisions are the right ones for operations.

A lesson learned during this initial phase was the support of too many simultaneous activities is not worth. Many times 4 till 5 activities in parallel were assisted by the GCT. The great disposition of the GCT and wiliness to help doing more activities as allowed - because it was required by other teams - produced more mistakes than success. The whole Columbus ground segment was planned by ESA to support a maximum of 2 activities in parallel, due to the availability of the systems and especially because of the reduced personal of the GCT. It is worth to be mentioned that each subsystem has only 1.5 SSE, 2 GOPs and 2 GOMs to support all the activities.

That situation produced an overload of the people producing mistakes in the configuration of the different systems. Some of them had a reconfiguration time of 45 minutes and was no possibility to check the systems for the upcoming activity after the reconfiguration. That caused a lot of blaming to the GCT and unnecessary stress and too long working days. For this reason the GOP and GOM decided to go back to the officially required and human possible support of two activities simultaneously. After heavy pressure of the GOMs, the numbers of GC and Syscon positions were increased in order to be able to have two teams (4 till 8 persons) to support long simulations and real time at the same time.

One of the big issues also was the time spend in explanations to Astrium and ESA GS. That was a too ineffective way to work. It was decided to implement a new daily reporting tailored for the management. That improved the whole communications and leaves the GCT time to concentrate in the big migration challenge planned for 2010 as is described below. In general the teams at Col-CC, ESA and Astrium had a steep learning curve during these first two years. That was difficult at the beginning but the dealing with the issues was awesome improved.

\section{Ground Segment Migration}

After 7 years of using the control center in preparation and for the Columbus mission, the ground segment subsystems are all running out of maintenance contracts and reached already end of life. Most of the Hardware and Software companies will not provide more maintenance to any equipment starting at 2010. That means a complete migration of the control center must be carried out. Such migration must be done in parallel to the normal activities, like real time operations, simulations (very time consuming) and intensive testing. All that should not be (longer) interrupted.

The negotiations for the refurbishment started end of 2008, but because of ESA GS budget cuts, and the complicated and bureaucratic structure, between DLR, Astrium and ESA, almost no agreement was arranged till beginning of 2010. That is producing delays and put the stability of the control center in a difficult situation. The voice system is running without maintenance contract, the SAN (Storage area network) is under the same situation. All the computers in the control and support rooms are out of life, without spare parts availability. The application servers of almost all the applications are in the same situation, video equipment is out dated. That means that the operations of the control center are under a high risk of being stopped if any medium failure takes place. Another important point is that all the applications must to be migrated to Sles 10 Linux and Windows 2008, which means not only new hardware, but also new operating system, new software versions and a huge test campaign.

In order to accelerate the long bureaucratic process and continue supporting operations, DLR decided to rent an equipment that represent a 3er part of the future SAN in order to start to test the different software upgrades. The 
most important limitations of the current SAN are the cycle 13 transition support software that can not run on the current operating system, the current operating system end of support and the SAN itself, that is out of life and without support. The current SAN was designed by ESA GS in a so redundant and secure way that now is impossible to make any operating system update, that means, no new software can be installed. The idea of the SAN NG (SAN new generation) is to keep the redundancy and security of the old SAN, but gives also the possibility to make updates and also to allow running different operating systems in parallel and simultaneously.

The SAN-NG (Storage area network new generation) migration could be done independently of the Col-CC Windows 2008, SLES10 (Linux operating system) migration as well as the new voice system. But the planning for Windows 2008, SLES 10 and SAN-NG testing are coordinated together for the following reasons:

- Testing of the DaSS SLES 10 version can only be done with the SAN-NG test-bed (rented equipment)

- To optimize the use of the subsystem engineering resources

- The TEST instance of the DaSS (Data Archiving subsystem) which is planned to be used for both of these activities is also used operationally so this planning needs to minimize the impact on the FCT.

Further, although MCS (monitoring and Commanding System) can be migrated to SLES10 using the newly introduced LUN setup (logical Unit on the SAN) this planning also includes testing MCS SLES 10 on the SAN-NG test-bed to support the SAN-NG proof-of-concept, once again for the last 2 reasons listed above.

Other subsystems covered as part of the overall Windows 2008, SLES 10 and SAN-NG testing are: SLES 10 Migration

- IMS (integrated manager subsystem)

- TQVS (Columbus simulator), strongly related to MCS and DaSS

Windows 2008 Migration

- MVDS (Mpeg video distribution system)

- OST (operation support tools)

Others

- Video hardware and software

- Network components ( switches, Routers and firewall exchange campaign already started end of 2009)

- Voice (a Move/Frequentis system)

- All PC's in operations and support rooms

In order to carry out all these activities and to minimize the impact on operations, an enormous coordination effort is needed. According to the current planning, if all the prerequisites are meet, like new SAN, new NIS (network integrated system), new WAN (wide area network), new voice, new software versions, new workstations and new application servers, only lost of redundancy will happened and some minutes of service interruptions. The mayor impact will be for the FCT and SIM teams, that will need to rotate a few times trough the 3 control rooms (K3, K4 and K11) in order to allow the refurbishment for the new voice system and WS in the control rooms, as well the test and validation activities of the new versions of central applications, like DaSS and MCS

It is NOT possible to migrate the DaSS on the current SAN related configurations, but all DaSS server also need simultaneous access to the Long-Term-RT-Archive (or at least the ones reading from the archive) which is not supported by a SAN-LUN configuration. Therefore, the only possible migration which can be performed currently is to migrate the DaSS TEST instance to SLES 10 and MCS SLES10 V4.0.x and then test the setup with SAN-NG Test Bed.

Once the SLES 10 testing is complete with the SAN-NG test-bed the DASS TEST instance will not be migrated back to the current SAN. The DaSS TEST SLES10 configuration will remain until the operational SAN-NG is available. The DaSS SLES10 long-term planning is dependent on SAN-NG implementation planning as without a SAN-NG the DaSS can not be migrated. In the short-term planning only the DaSS kernel SLES 10 migration local testing is taken into account. However, for the long-term planning the SLES 10 migration needs to also take into account migration of remote DaSS components i.e. the DaSS DEMUX located in HOSC and the TEST DEMUX 
located in Col-ESC Bremen. These components are not dependent on the SAN implementation and therefore can be independently planned.

The IMS Prime Set-Up is currently configured as Semi-SANless. The IMS binaries are stored on local discs on the IMS application servers and the IMS database is stored on the SAN mount point. The IMS Prime consists of 7 SAN servers. The IMS - in a similar manner to DaSS - cannot be migrated to SLES 10 without SAN-NG. The reason for that is the IMS database which is based on the failover capability of mount points of SAN servers.

\section{H. Client migration}

For all the control rooms and in order to support the SLES 10 operating system and because all the clients already are over the end of life, new computers must be installed. A test campaign with all the new versions of the applications were carried out during 5 months, using different hardware configurations. The clients will be have hard disk with a low speed and in a Raid 5 configuration with the possibility to be hot exchanged without interruptions in operations, reducing the current repair time of almost one working day. When the clients will be finally acquired, a good planning with current operations must be re-planned (already 2 months delay) with all the subsystems, trying to reduce the drop off times of the different control room and the associated support rooms

\section{Partial video migration}

Tests installing the Windows 2008 operating system on the iCue servers failed. According to IBM the current hardware does not support Windows 2008. Using currently available hardware at Col-CC a Windows 2008 based iCue version will not be available in 2009. ICue will stop supporting Windows 2000 based versions in the second quarter of 2010. Tests with a new server started at the beginning of 2010, if the second one is available on time the migration of the redundant video system will be an interruption of a few seconds. The complete video migration discussion started middle of 2009, but it was decided to wait till 2011 to carry out this migration due to ESA money restrictions.

\section{J. OST}

Must upgrade servers and install Windows 2008. New versions of NASA software will require .Net 3.5 which will only be supported on Windows 2008. As the current server hardware does not support Windows 2008, new hardware needs to be procured before the start of the migration to Windows 2008. This migration can be done in parallel to the control rooms refurbishment, but the servers must be available at the same time as this task take place in order to have time to test it and not interrupt operations.

\section{K. MCS SAN-NG Testing}

That will be first test performed using the MCS SLES8 version to have a known behavior/status of the system when testing on the SAN-NG test-bed. It has been generally agreed that is does not make sense to test with several significant changes at once i.e. a new system with SLES 10, a new SLES10 software release and a new SAN. Due to the successful testing of the new MCS in the SAN test bed, the new version using SLES 10 servers and clients is being tested. The MCS software is the principal driver for the Linux migration and the movement of the teams to the different control rooms.

\section{Voice Migration}

After many discussions, it was finally decided that Col-CC will have a voice system compatible with NASA. The same company that builds the MOVE system for NASA was contacted in order to have the same system at ColCC. Many technical discussions took place in order to adapt the keyset to the "European" format, actually one of the 3 standards of the company. Because of the many delays caused by the discussions with ESA GS, the voice system will be not in time to be installed in parallel with the control rooms refurbishment. The Voice system is the most important migration at Col-CC, the transition to the external and internal systems will be done step by step. First the new matrix will be installed and some loops will be migrated till the FCT is comfortable with the new system and keysets and finally in one step the complete system will be connected to the new one, causing only some minutes of interruptions. The main issue here is that the new system uses other cables than the current ones and that must be physically exchanged in the rooms.

The whole migration planning, coordination with other teams and implementation is being carried out with the same personal, no new positions are foreseen to carry out this huge task, that means a lot of extra working hours, good will and cooperation of the existing personal is the only way to realize this task and continue in parallel with normal operations and maintenance. This whole project in only possible because great support of the Col-CC staff. 


\section{Project Risks}

Currently due to the many delays caused by the ESA GS bureaucracy the whole migration will be delayed and control center is running in a very delicate situation. In this section a summary of the different subsystems and their risks is presented.

9. DaSS: Two options for the migration of the DaSS Ops to SLEs 10 are possible.

First, order 10 new servers and use them to build a new DaSS Ops. During the actual SLES 10 migration of the DaSS Ops, the current DaSS Ops servers will simply replaced using the new servers. During the entire installation period of the current DaSS Ops, the DaSS Test and DaSS Sim will be available to support simulations (ESs, JMSTs, and ATV JISs).

Second, the migration of DaSS to SLES 10 will also be possible without the 10 new servers. In this scenario both the DaSS Test and DaSS Sim servers will be used to build the new DaSS Ops. The migration between the current SLES 8 DaSS Ops and the new SLES 10 DaSS Ops will be performed by swapping the old servers with the newly installed ones. Using this approach Col-CC will not be able to support any simulations (ESs, JMSTs, or ATV JISs) for about two months while the new DaSS Ops is being installed on the current DaSS SIM and Test servers.

This very important point that can delay the whole simulations and training schedule is still under discussion since several months with ESA GS, the cost of the meetings is already higher than the cost of the servers, but not decision was taken till now. Col-CC is quite confidence that these servers will be acquired because the impact is just too high to be acceptable compared to the very low cost of this option.

In case of the 10 additional DaSS servers for parallel setup and testing are acquired, 6 of the former, now spare, servers could be used for temporary replacement of the HOSC demux servers that are in a critical status.

10. HOSC Demux: The demux servers in HOSC are reaching their end-of-life with no available spares in HOSC. Replacement of the servers is needed. Failure of the existing hardware will result in impacts to the ongoing operations. The proposal was to order six new servers as replacement. Three server pairs, each pair would be used for demuxes for real-time, external playback and PDSS requests. Additionally the demux servers in HOSC need to be upgraded to SLES 10. Having additional servers in place will allow the installation and configuration parallel to the ongoing operations and will only cause minor outages while the hardware is swapped.

A migration to SLES 10 will be possible using the existing hardware but as there are only 1.5 fully operational demuxes available in HOSC the demux being upgraded will be offline for about four days during the installation process. This will cause an impact to operations as only one demux for real time or external playback will be available (no PDSS requests). Two of the servers are already out of service and a $3^{\text {rd }}$ one is having hardware problems that means the whole KU band traffic to Europe can be interrupted at any time. This important point is also still under discussion since several months.

11. MCS: The 9 additional servers for MCS would be used to build a redundant MCS instance for K3 (control room 3). The advantages would be an outage of the MCS K3 instance of maximum two weeks compared to two months as the servers could be installed and configured offline. But more important having the redundant MCS instance will allow for failover testing on the MCS 4.0.X at the beginning of the migration period. Not having these 9 servers will only have minor impact to the migration process, but the K3 instance of MCS will not be redundant and therefore failover testing can only be performed after the migration of $\mathrm{K} 4$ at the end of the migration period. Finding issues on the failover algorithms at that late date can cause serious impacts to the ongoing operations. Till now is not decision to acquire these servers. Big delays must be planned in the main control room K4 with consequences in the SIM schedule.

12. OST: as mentioned, after tests on October 20, 2009 it was confirmed that the current server hardware will not support the installation of Windows 2008. NASA will stop their OST tools support for Windows 2000/2003 in June 2010. Not providing the new server hardware will result in a loss of the NASA OST tool after their migration to Windows 2008. No decision regarding this point is taken till now. 
13. MVDS (iCue): As for OST above the current iCue server hardware will not support the installation of Windows 2008 and iCue will stop supporting the Windows 2000 version of their software in the second quarter of 2010. As the current hardware does not support Windows 2008 not upgrading will result in no support from iCue until ColCC migrates iCue to the Windows 2008 version. This software is used to select the inputs and outputs of the video system in Col-CC and to the external sites

14. VoCS: as mentioned before the current voice system reached of the end of life and no maintenance contract exists for 2010. Only a few old spare parts exist at Col-CC and the IP. A failure of the system will be imply the total or partial lost of communications with probably days or weeks of interruptions, depending the nature of the failure. This is an extreme critical situation where ESA Ground Segment must to speed up with the decisions in order to warranty the operations of Columbus. Col-CC is pretty sure that this issue will be solved soon.

\section{Conclusion}

Resuming the content of the paper, the history of the delays and many configuration changes of the Columbus project is presented. A relative short description of the many functions of the ground control team, as well as the job of the different members and the interaction with the others teams working at the Columbus and ATV projects are also presented.

The history of failures and the effectiveness of the ground control team to solve them and continue supporting operations is reported

A description of the urgently needed migration of the whole control center infrastructure is described, together with the high risks that the control center in running into due to the many delays in the decisions to proceed with the required updates. The risk of the delays and a short description of the migration of the different subsystems are also described.

\section{Acknowledgments}

At this point I would like to especially acknowledge the Ground Control Team at the Col-CC for their unconditional support and their constant willingness to provide everything necessary to make the mission a success. 


\section{Appendix A: Glossary}

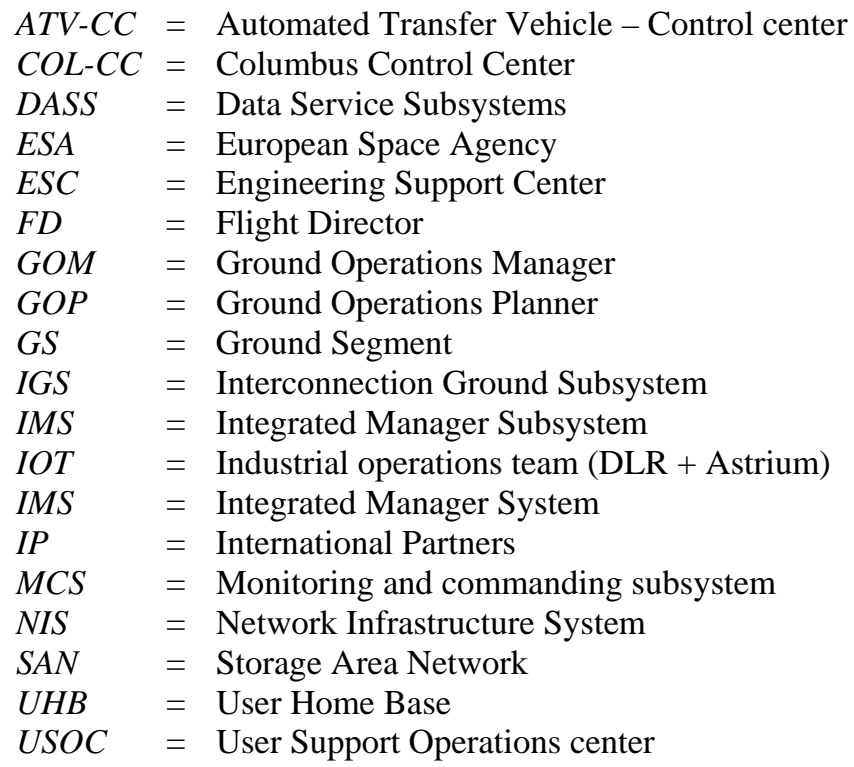

